\title{
Pathological Findings and Immunohistochemical Evaluation of MMP-2 and TIMPs in Equine Fetlock Affected by Degenerative Joint Disease
}

\author{
Redento Mora ${ }^{1}$, Diana Binanti ${ }^{2}$, Nicoletta Mora ${ }^{2}$, Eleonora Fantinato ${ }^{2}$, Valentina Ferrante ${ }^{2}$, \\ Luisella Pedrotti ${ }^{1}$, Pietro Riccaboni ${ }^{2}$ \\ ${ }^{1}$ Orthopaedic Section; Department of Surgical, Diagnostic and Pediatric Sciences, University of Pavia, "Città di Pavia Institute" University \\ Hospital, School of Medicine, Pavia, Italy \\ ${ }^{2}$ Department of Veterinary Science and Public Health, University of Milan, Veterinary University Hospital, School of Veterinary Medicine, \\ Lodi, Italy
}

\section{Email address:}

tinomora@hotmail.com (R. Mora),diana.binanti@unimi.it (D. Binanti), nicolettamora@hotmail.com (N. Mora), eleonora.fantinato@unimi.it (E. Fantinato), valentina.ferrante@unimi.it (V. Ferrante), luisella.pedrotti@tiscali.it (L. Pedrotti), pietro.riccaboni@unimi.it (P. Riccaboni)

\section{To cite this article:}

Redento Mora, Diana Binanti, Nicoletta Mora, Eleonora Fantinato, Valentina Ferrante, Luisella Pedrotti, Pietro Riccaboni. Pathological Findings and Immunohistochemical Evaluation of MMP-2 and TIMPs in Equine Fetlock Affected by Degenerative Joint Disease. American Journal of Clinical and Experimental Medicine. Vol. 3, No. 4, 2015, pp. 172-177. doi: 10.11648/j.ajcem.20150304.18

\begin{abstract}
Background: MMP-2 and MMP-9 are proteolytic enzymes involved in the remodeling of extracellular matrix and regulated in their function by a family of proteins called TIMPs. MMP-2 and MMP-9 and TIMPs are secreted in joints by both synovial cells and chondrocytes, and are implicated in osteoarthritis onset and progression. The aim of this study was to investigate the localization of MMP-2 and TIMPs expression and their relationship in the synovial membrane of equine fetlock (metacarpo-phalangeal joint), which is the joint most frequently affected by osteoarthritis due to his high weight-bearing impact and shearing forces. Method: This study analyzed the immunohistochemical expression of MMP-2 and tissue inhibitors of MMP (TIMPs) in synovial membrane sections from 40 equine metacarpo-phalangeal joints affected by osteoarthritis in order to better assess their role in the pathogenesis of joint-structure alterations. Immunohistochemical study was performed using the Avidin-Biotin-Peroxidase complex method, and the percentage of labeled cells was assessed semi-quantitatively. Data obtained were statistically revised through the Spearman's rank correlation coefficient. Results: MMP-2 and TIMPs were expressed by type-B synoviocytes and endothelial cells. MMP-2 expression was not significantly associated to the macroscopic findings while a linear correlation between MMP-2 and TIMPs expression was observed. Conclusion: These data suggest that in degenerative joint disease the synovial expression of TIMPs may contribute to the regulation of MMP-2 expression in order to reach a new dynamic balance and that MMP-2 plays an important pro-homeostatic role in physiological and pathological equine synovial membrane.
\end{abstract}

Keywords: MMPs, TIMPs, Fetlock, Horse, Immunohistochemistry

\section{Introduction}

Matrix metalloproteinases (MMPs) are a large family of zinc-dependent endopeptidases involved in physiological turnover mechanisms and in the pathological degradation of extracellular matrix (ECM). Loss of articular cartilage ECM, a dynamic network of interacting molecules such as collagens, fibronectin, laminin and proteoglycans [1], is central to the pathogenesis of diseases such as osteoarthritis [2].
MMPs are mostly expressed by mesenchymal cells including fibroblasts, chondrocytes, osteocytes and inflammatory cells such as macrophages, leucocytes and plasma cells [3].

To date, at least 24 MMPs expressed in human tissues have been identified; they have been classified into five families: collagenases, gelatinases, stromelysins, matrilysins and 
membrane-type MMPs [3, 4].

MMPs participate in physiological processes (such as embryonic development, tissue remodelling and skeletal growth) and pathological events (like inflammatory processes, cardiovascular and autoimmune diseases, neoplasia and degenerative joint diseases) [5 - 9].

MMPs activity is regulated by several inhibitors, mainly the tissue inhibitors of metalloproteinases (TIMPs) produced by a variety of cell types and tissues [10]. Four TIMP families have been identified (TIMP-1, TIMP-2, TIMP-3, TIMP-4) [1]. They play a key role in balancing ECM deposition and degradation and they regulate several physiological and pathological processes such as angiogenesis, tumour cell invasion and wound healing [11].

In humans, the pathogenetic role of specific MMPs, such as MMP-2, MMP-9 and MMP-13, has been recently investigated in different joint diseases such as rheumatoid arthritis and osteoarthritis (OA) $[8,9,12]$. Aberrant regulation, resulting in an excess of MMPs over TIMPs, is thought to be a key event in the transition from physiological to pathological joint conditions [6].

In Veterinary Medicine, especially concerning the horse, several studies assessed the role of MMPs and their TIMP inhibitors in diseases such as keratitis, laminitis and pulmonary disease $[13,14,15]$.

Osteoarthritis, also known as degenerative joint disease, is the most common articular disease affecting humans and several animal species, especially horses and dogs. It is a chronic, degenerative process characterised by progressive cartilage erosion, subchondral bone remodelling, osteophytosis, synovial membrane thickening and consequent loss of joint function [16]. Although its primary cause is still unknown, it has been demonstrated that its development is associated with a cascade of biochemical events mediated by cytokines, i.e. IL-1, IL-6, proteolytic enzymes and other pro-inflammatory molecules; these events, together with the increased synthesis of MMPs, will upset the dynamic balance of cartilage ECM and lead to pathologic alterations.

Equine fetlock (metacarpo-phalangeal joint) and carpus are the joints most frequently affected by osteoarthritis due to their high weight-bearing impact and shearing forces [16].

Although there is little data available on the correlation between MMPs and degenerative joint disease in horses, some studies have exploited the gelatin-zymography technique and demonstrated the presence and the activity of TIMPs [2] and gelatinases A (MMP-2) and B (MMP-9) in synovial fluids [17, $18]$.

In almost all tissues MMP-2 is produced mainly by fibroblasts, endothelial and epithelial cells. MMP-2 is above all a homeostatic factor: in reference to rheumatoid arthritis in humans, many findings concur in defining a suppressive role of the enzyme, supporting its systemic pro-homeostatic function. MMP-2 is also associated with pathological processes, such as cardiovascular and neoplastic diseases [3, 19], where it is strictly correlated to tumourigenesis especially in the phases of neovascularisation and cellular invasion. MMP-2 is aberrantly up-regulated in the tumour microenvironment and its elevated serum concentration makes it a relevant diagnostic and prognostic negative marker.

MMP-9 production by fibroblasts, keratinocytes, chondrocytes, osteoblasts and endothelial cells is highly correlated to inflammatory events; its involvement has been described in neoplastic, cardiovascular and auto-immune diseases [3].

This study aims to investigate the immunohistochemical expression of MMP-2 and TIMPs in the synovial membrane of metacarpo-phalangeal joints of horses affected by degenerative joint disease and to better assess their role in the pathogenesis of joint-structure alterations.

\section{Methods}

\subsection{Case Selection}

At a slaughterhouse, 40 metacarpo-phalangeal joints of horses apparently affected by fetlock disease were selected. No data regarding sex, age or breed of the animals were available. Macroscopic, histological and immunohistochemical examinations were performed on each joint.

\subsection{Gross Pathology}

Bone, cartilage and synovial membrane were examined to identify any macroscopic alterations. Findings were scored on a scale from 0 (= no alterations) to 3 (= severe alterations), depending on the type and extent of the lesions. Synovial tissue of an adult, healthy horse - with neither macroscopic nor microscopic lesions - was used as negative control.

\subsection{Histopathology}

A total of 3 samples from the fetlock synovial recesses (dorsal, palmar lateral, palmar medial) were collected from each joint, for a total of 120. Samples were fixed in $10 \%$ neutral buffered formalin for up to 72 hours. Samples were embedded in paraffin wax and $4 \mu \mathrm{m}$ thick sections were stained with haematoxylin and eosin.

\subsection{Immunohistochemistry (IHC)}

The 120 samples were examined for MMP-2 expression. In reference to TIMPs, only one sample per joint, for a total of 40 specimens, was evaluated.

Formalin-fixed, paraffin-embedded tissue sections were examined immunohistochemically using the Avidin-Biotin-Peroxidase complex method (Vectastain Elite ABC kit, Vector Laboratories, Burlingame, CA). Tab.1: eliminated as requested by reviewer. $\leftarrow$ ELIMINATE!!!!!!!

For antigen retrieval, sections were treated with citrate buffer at $\mathrm{pH} 6.0$ at a temperature of between $95^{\circ} \mathrm{C}$ and $100^{\circ} \mathrm{C}$, maintained for 10 minutes. Peroxidase activity was investigated with a solution of 3,3-diaminobenzidine tetrahydrochloride (DAB) at room temperature. Counterstaining was performed with Mayer's haematoxylin.

The percentage of labeled cells was assessed 
semi-quantitatively and scored as follows: -, absence of labeled cells;,$+<30 \%$;,++ 30 to $60 \% ;+++$, more than $60 \%$.

\subsection{Statistical Analysis}

Data obtained were statistically revised through the Spearman's rank non parametric correlation coefficient (Spearman rho; SPSS 18.0).

Results were considered statistically significant at $\mathrm{P}<0.05$. SPSS 18.0 was used to evaluate: 1 ) the correlation between the expression of MMP-2 and TIMPs, 2) the correlation between macroscopic and immunohistochemical results.

\section{Results}

\subsection{Gross Pathology}

Less damaged joints were classed as Grade 1 (Fig. 1) and presented widespread thinning of cartilage, a condition generally associated to mild chondromalacia. Patterns of cartilage remodelling associated with severe chondromalacia were frequently found and classed as Grade 2 (Fig. 2).

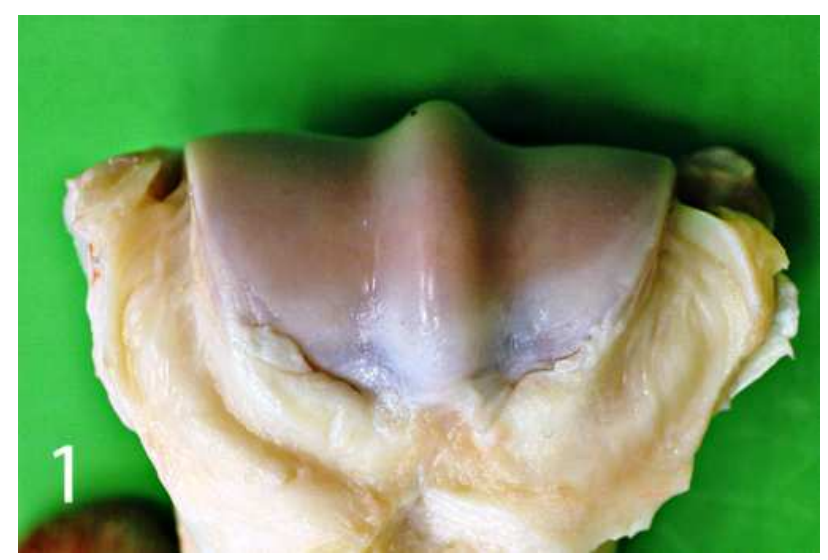

Figure 1. Horse degenerative joint disease grade 1. Articular surface showed diffuse thinning of cartilage.

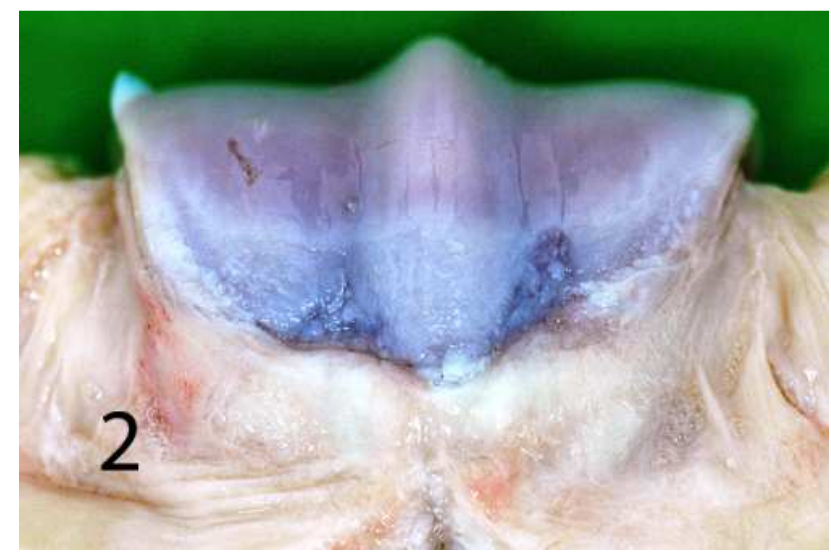

Figure 2. Horse degenerative joint disease grade 2. Articular surface showed longitudinal linear grooves and multifocal moderate chondromalacia.

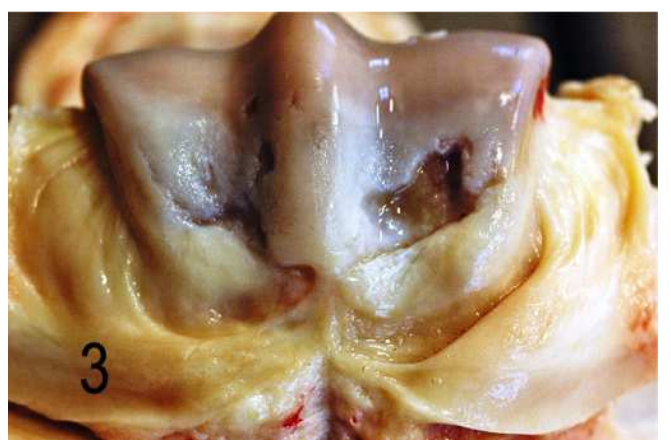

Figure 3. Horse degenerative joint disease grade 3. Articular surface showed severe diffuse chondromalacia, multifocal loss of cartilage with exposure of subchondral bone, associated to marginal osteophytes.

In more severe cases, cartilage showed deep longitudinal grooves with multiple erosions associated to marginal or intra-articular osteophytes: these joints were classified as Grade 3 (Fig. 3).

Of the 40 joints examined, eight joints were macroscopically evaluated as Grade 1, 13 joints as Grade 2 and 19 joints as Grade 3. None of the joints examined was classified as Grade 0 . The joint used as negative control was classed as Grade 0 .

\subsection{Histopathology}

All specimens showed similar histological patterns. Mild lymphocytic infiltration of synovial stroma with scattered lymphocytes sporadically organized in small follicle-like structures were observed in most histological specimens. Mild perivascular fibrosis and moderate oedema were also present.

\subsection{Immunohistochemistry}

MMP-2 immuno-labelling. In at least one of the three recesses, MMP-2 immuno-labelling was present. MMP-2 was expressed by Type-B synoviocytes and endothelial cells, and cytoplasmic labelling was always observed.

In 42 samples, no MMP-2 immuno-labelling was detectable. The remaining samples showed a grade + MMP-2 expression in 33 (Fig. 4), ++ in 33 (Fig. 5), +++ in 12 cases respectively (Fig. 6). The MMP-2 expression of the control specimen was classified as +++ .

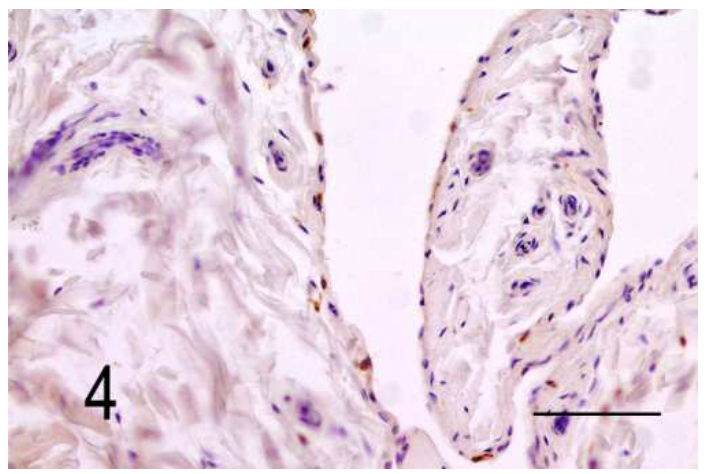

Figure 4. In 33 out of 120 samples evaluated, MMP-2 immunolabelling was expressed by type-B synoviocytes and endothelial cells in less than $30 \%$ of cells (Grade + MMP-2 expression) (IHC, DAB, hematoxylin counterstain, Bar $=150 \mu \mathrm{m})$. 


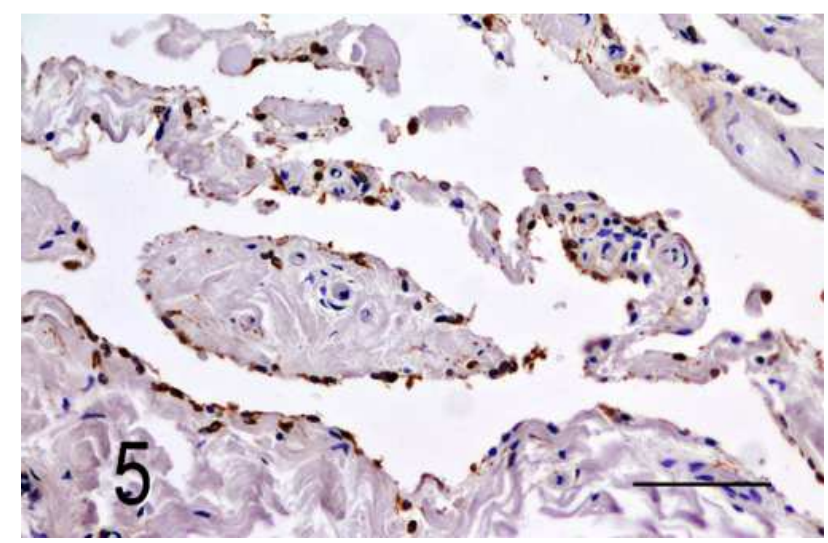

Figure 5. In 33 out of 120 samples evaluated, MMP-2 immunolabelling was expressed by Type-B synoviocytes and endothelial cells in less than $60 \%$ of cells (Grade ++ MMP-2 expression) (IHC, DAB, hematoxylin counterstain, Bar $=150 \mu \mathrm{m})$

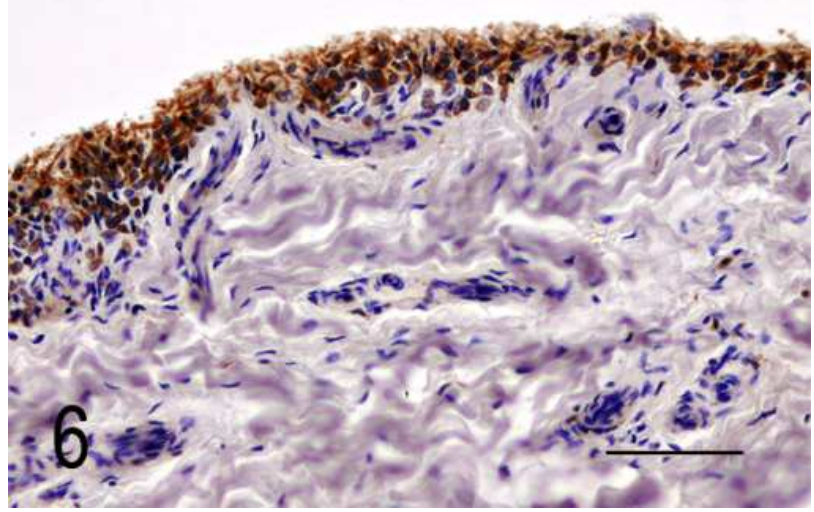

Figure 6. In 12 out of 120 samples evaluated, MMP-2 immunolabelling was expressed by Type-B synoviocytes and endothelial cells in more than $60 \%$ of cells (Grade +++ MMP-2 expression) (IHC, DAB, hematoxylin counterstain, Bars $=150 \mu \mathrm{m})$.

TIMPs immune-labelling. TIMPs were expressed in the cytoplasm of type-B synoviocytes and of endothelial cells of 30 fetlocks. In 16 cases, immuno-labelling was classified as + (Fig. 7), 12 as ++ (Fig. 8) and 2 as +++ (Fig. 9). Samples from 10 cases and the negative control showed a total absence of TIMPs expression. The results are shown in Table 1.

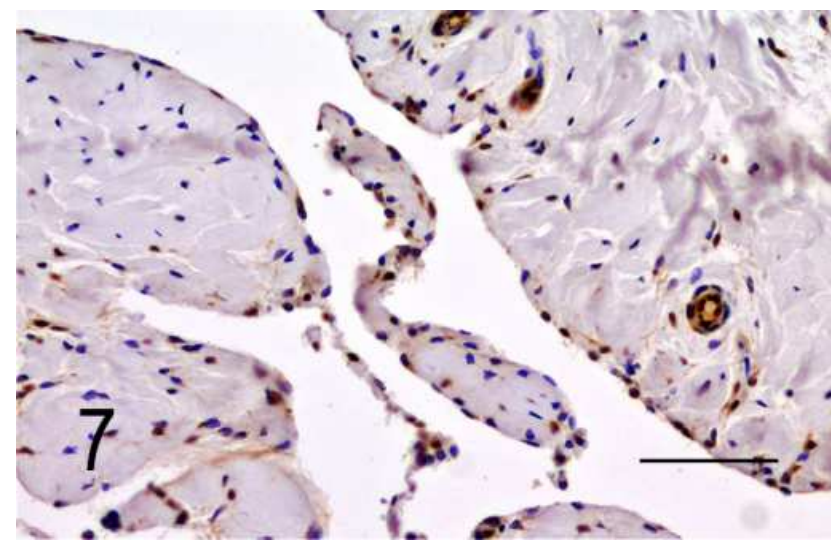

Figure 7. In 16 out of 40 samples evaluated, TIMPs immunolabelling was expressed by Type-B synoviocytes and endothelial cells in less than $30 \%$ of cells (Grade + TIMPs expression) (IHC, DAB, hematoxylin counterstain, Bars $=150 \mu \mathrm{m})$.



Figure 8. In 12 out of 40 samples evaluated, TIMPs immunolabelling was expressed by Type-B synoviocytes and endothelial cells in less than $60 \%$ of cells (Grade ++ TIMPs expression) (IHC, DAB, hematoxylin counterstain, Bars $=150 \mu \mathrm{m})$.

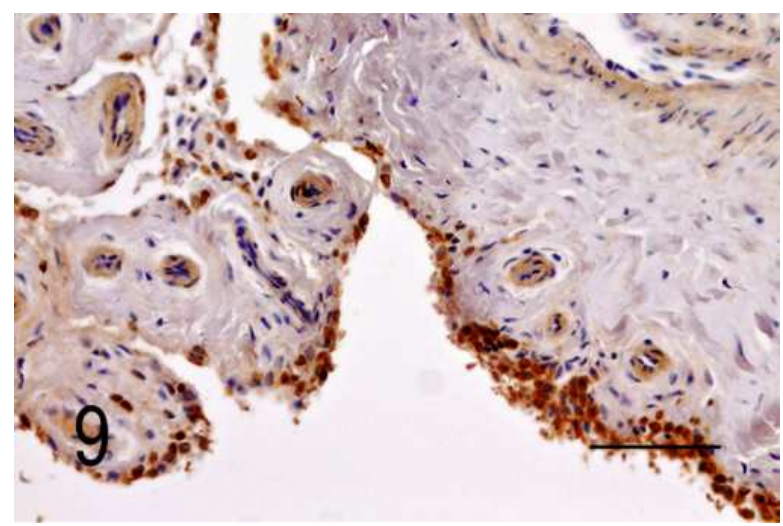

Figure 9. In 2 out of 40 samples evaluated, TIMPs immunolabelling was expressed by Type-B synoviocytes and endothelial cells in more than $60 \%$ of cells (Grade +++ TIMPs expression) (IHC, DAB, hematoxylin counterstain, Bars $=150 \mu \mathrm{m})$.

\subsection{Statistical Analysis}

Statistical analysis showed a strong positive correlation between MMP-2 and TIMPs $(\mathrm{P}<0.004)$, while no correlation between macroscopic and immunohistochemical examination was found.

Table 1. Immunohistochemistry for matrix metalloproteinasis 2 (MMP-2) and their inhibitors (TIMPS).

\begin{tabular}{|c|c|c|c|c|c|c|c|c|}
\hline & \multicolumn{4}{|c|}{ MMP-2 (\%) N=120 } & \multicolumn{4}{|c|}{ TIMPs (\%) $N=40$} \\
\hline & 0 & + & ++ & +++ & 0 & + & ++ & +++ \\
\hline M & $14(35)$ & $13(32$ & $8) 8(20)$ & $5(12.5)$ & $5(38.4)$ & $4(30.7)$ & $3(23)$ & $1(7.6)$ \\
\hline $\mathrm{L}$ & $5(12.5)$ & $12(30$ & ) $19(47$ & 5)4 (10) & $1(7.1)$ & $6(42.8)$ & $6(42.8)$ & $1(7.1)$ \\
\hline D & $23(57.5)$ & $8(20)$ & $6(15)$ & $3(7.5)$ & $4(30.7)$ & $6(46.1)$ & $3(23)$ & 0 \\
\hline
\end{tabular}

3 samples from the fetlock synovial recesses were collected from each joint (D: dorsal, L: palmar lateral, M: palmar medial). Immuno-labelling was classified semi-quantitatively and scored as follows: 0 , no labeling;,$+<30 \%$; ,++ 30 to $60 \% ;+++$, more than $60 \%$.

\section{Conclusions}

Several studies investigated the up-regulation of MMPs and TIMPs in OA in human beings as well as in animals [14, 20, 
21]. Previous reports assessed that local MMPs chondrocyte production in OA cartilage varies with the extent of cartilage damage [22]. However, the mechanisms of MMPs regulation and their intra-articular distribution are still unclear.

The main aim of this study is to investigate the immunohistochemical distribution of MMP-2 expression in the synovial membrane of equine fetlock affected by OA.

Many authors have investigated MMPs in horse synovial fluid employing different technical approaches, i.e. zymography; they focused their attention on MMP-2 and MMP-9 activity in joint diseases $[2,17,18,20]$. These studies demonstrated the high presence of MMP-2 and MMP-9 in the synovial fluid of pathological joints.

In this study, the expression of MMP-2 in the articular soft tissue was restricted only to Type-B synoviocytes and endothelial cells.

These findings agree with literature data that indicate this as a constant in human patients [3]. MMP-2 in fact, is expressed by several cell types, including Type-B synoviocytes, both in healthy joints, as shown by the negative control, and in pathological ones, as demonstrated by samples affected by degenerative joint disease.

In a previous study [22] concerning MMP-2 immuno-staining performed on human temporomandibular fibrocartilage, researchers identified a linear correlation between MMP-2 positive cell rate and macroscopic carti-lage damage.

In this study, the immuno-labelling was observed in at least one of the investigated articular recesses but no statistical correlation with the severity of anatomical lesions was found.

This finding is apparently contradictory; however it must be emphasized that MMP-2 appears to have a dual activity. This enzyme is involved in matrix degradation and ECM remodelling, contributing to disease progression; on the other hand, experimental findings demonstrate that high concentrations of MMP-2 are a consequence of the efforts to down-regulate the inflammatory stimulus, confirming the pro-homeostatic role of MMP-2 [3].

The lack of relationship between macroscopic and immunohistochemical examinations can also be explained by the characteristics of the disease. All the joints examined in this study were in fact affected by lesions of different type, extent and severity; however the degenerative disease was always chronic. Elevated levels of MMP-2 in serum and in synovial fluid were characteristically detected in patients in an advanced stage of the disease [3].

The chronic process seems to reach a condition of "pathological homeostasis", with substantial equilibrium of antigenic expression, independent of the original anatomical lesions.

MMP-2 activity is tightly-regulated by TIMPs, especially TIMP-2, TIMP-3 and TIMP-4, that display relevant affinity for this MMP [3]. For this reason an antibody that recognises all TIMPs molecules has been employed.

This study demonstrates a significant correlation between MMP-2 and TIMPs expression in all samples. TIMPs immuno-labelling was observed in most of pathological samples, but was absent in the negative control. These findings are in agreement with previous studies performed with zymography of fetlock synovial fluid [2].

Lack of TIMPs expression in normal fetlock synovial membrane observed in this study may represent the physiological balance between MMP-2 and its regulators. Under physiological conditions MMP-2 is expressed and probably does not require the inhibiting activity of TIMPs. Moreover, under pathological conditions, the synovial expression of TIMPs may contribute to the regulation of MMP-2 expression and activity in order to reach a new "dynamic balance", suggesting that TIMPs may also have a fundamental role in joint homeostasis. In fact, the imbalance between MMPs and TIMPs leads to ECM degradation and consequently to articular damage [22].

This is the first study that describes immunohistochemical expression of MMPs and TIMPs in synovial membrane of horses affected by OA. Chondrocytes, synoviocytes and inflammatory cells infiltrating synovial membrane have been identified as being the MMP-producing cells in humans [22].

This study demonstrated the expression of MMP-2 by type-B synoviocytes in horse fetlocks, under both physiological and chronic pathological conditions. Expression of MMP-2 has been investigated in human medicine in chondrocytes, confirming its important role in fibrocartilage degradation. Further studies regarding chrondrocyte immunohistochemical expression of MMP-2 and TIMPs may provide more information on their mechanisms and role in degenerative joint diseases.

The resulting data (positive correlation between MMP-2 and TIMPs expression; no correlation between the macroscopic findings and MMP-2 expression) suggest that under pathological conditions, the synovial expression of TIMPs may contribute to the regulation of MMP-2 expression and activity in order to reach a new "dynamic balance", and that MMP-2 plays an important pro-homeostatic role in physiological and pathological equine synovial membrane.

\section{References}

[1] Boveland S., Moore P., Mysore J., Krunkosky T.M., Dietrich U.M., Jarrett C., Paige Carmichael K.: Immunohistochemical study of matrix metalloproteinases- 2 and -9 , macrophage inflammatory protein-2 and tissue inhibitors of matrix metalloproteinases-1 and -2 in normal, purulonecrotic and fungal infected equine corneas. Vet Ophtalmol 2010, 13: 81-90.

[2] Brew K., Dinakarpandian D., Nagase H.: Tissue inhibitors of metalloproteinases: evolution, structure and function. Biochim Biophys Acta 2000, 1477: 267-283.

[3] Carmona J.U., Prades M.: Pathophysiology of Osteoarthritis. Compendium Equine.com 2009, January/February: 28-40.

[4] Clegg P.D., Carter S.D.: Matrix metalloproteinase -2 and -9 are activated in joint diseases. Equine Vet J 1999, 31: 324-330.

[5] Clegg P.D., Coughlan A.R., Carter S.D.: Equine TIMP-1 and TIMP-2: Identification, activity and cellular sources. Equine Vet J 1998, 30: 416-423. 
[6] Clegg P.D., Coughlan A.R., Riggs C.M., Carter S.D.:Matrix metalloproteinases 2 and 9 in equine synovial fluids. Equine Vet J 1997, 29: 343-348.

[7] Clutterbuck A., Harris P., Allaway D., Mobasheri A.: Matrix metalloproteinases in inflammatory pathologies of the horse. Vet J 2010, 108: 27-38.

[8] Curran S., Murray G.I.: Matrix metalloproteinases in tumour invasion and metastasis. J Pathol 1999, 189: 300-308.

[9] Fassina G., Ferrari N., Brigati C., Benelli R., Santi L. Noonan D.M., Albini A.: Tissue inhibitors of metalloproteases: Regulation and biological activities. Clin Exp Metastasis 2000: 18: $111-120$.

[10] Fietz S., Einspanier R., Hoppner S., Hertsch B., Bondzio A.: Determination of MMP-2 and -9 activities in synovial fluids of horses with osteoarhritic and arthritic joint diseases using gelatin zymography and immunocapture activity assays. Equine Vet J 2008, 40: 266-271.

[11] Kevorkian L., Young D.A., Darrah C., Donell S.T., Shepstone L., Porter S. et al.: Expression profiling of metalloproteinases and their inhibitors in cartilage. Arthritis Rheum 2004, 50: 131-141.

[12] Kyaw-Tanner M.T., Wattle O., van Eps A.W., Pollitt C.C.: Equine laminitis: Membrane type matrix metalloproteinase-1 (MMP-14) is involved in acute phase onset. Equine Vet $\mathrm{J}$ 2008,40: 482-487.

[13] Lambert E., Dassé E., Haye B., Petitfrère E.: TIMPs as multifacial proteins. Critical Rev Oncol Hematology 2004, 49: 187-198.

[14] Murphy G., Lee M.H.: What are the roles of metalloproteinases in cartilage and bone damage? Ann Rheum Dis 2005, 64:
44-47.

[15] Nagase H., Visse R., Murphy G.: Structure and function of matrix metalloproteinases and TIMPs. Cardiovasc Res 2006, 69: $562-573$.

[16] Sbardella D., Fasciglione G.F., Gioia M., Ciaccio C., Tundo G.R., Marini S., Coletta M.: Human matrix metalloproteinases: An ubiquitarian class of enzymes involved in several pathological processes. A review. Mol Aspects Med 2012, 33: 119-208.

[17] Tanaka A., Kawashiri S., Kumagai S., Takatsuka S., Narinobou M., Nakagawa, K. et al.: Expression of matrix metalloproteinase-2 in osteoarthritic fibrocartilage from human mandibular condyle. J Oral Pathol Med 2000, 9: 314-320.

[18] Tetlow L.C., Daman J.A., Woolley D.E.: Matrix metalloproteinase and proinflammatory cytokine production by chondrocytes of human osteoarthritic cartilage. Arthritis Rheum 2001, 44: 585-594.

[19] Van den Steen P.E., Dubois B., Nelissen I., Rudd P.M., Dwek R.A., Opdenakker G.: Biochemistry and molecular biology of gelatinase B or matrix metalloproteinase-9 (MMP-9). Crit Rev Biochem Mol Biol 2002, 37: 375-536.

[20] Visse R, Nagase H.: Matrix Metalloproteinases and Tissue inhibitors of Metalloproteinases: Structure, Function and Biochemistry. Circ Res 2012, 92: 827-839.

[21] Galasso O., Familiari F., De Gori M., Gasparini G.: Recent findings on the role of gelatinases (Matrix Metalloproteinases -2 and -9) in osteoarthritis. Adv Orthop 2012 ID 834208: 1-7.

[22] Murphy G., Nagase H.: Reappraising metalloproteinases in rheumatoid arthritis and osteoarthritis; destruction or repair? Nature Clin Pract Rheumatol 2008, 4: 128-135. 